\title{
On the Origins of the Special Theory of Relativity
}

\section{Citation}

Holton, Gerald. 1960. On the Origins of the Special Theory of Relativity. American Journal of Physics 28, 627. https://doi.org/10.1119/1.1935922

\section{Permanent link}

http://nrs.harvard.edu/urn-3:HUL.InstRepos:37902460

\section{Terms of Use}

This article was downloaded from Harvard University's DASH repository, and is made available under the terms and conditions applicable to Other Posted Material, as set forth at http:// nrs.harvard.edu/urn-3:HUL.InstRepos:dash.current.terms-of-use\#LAA

\section{Share Your Story}

The Harvard community has made this article openly available.

Please share how this access benefits you. Submit a story.

Accessibility 


\title{
On the Origins of the Special Theory of Relativity*
}

\author{
Gerald Holton \\ Department of Physics, Hiarvard University \\ (Received May 9, 1960)
}

\begin{abstract}
Einstein's early work on relativity theory is found to be related to his other work at that time (e.g., in subject matter and style). In addition to this element of internal continuity one finds also-as a key to a new evaluation of the significance of Einstein's contribution-an external continuity with the classic, Newtonian tradition governing restrictions on permissible hypotheses. On the other hand, Einstein's work is shown to have been, in important respects, more independent of other contemporary work in this field than has recently been proposed.

These continuities and discontinuities are set forth to make the point that philosophical studies of scientific work should proceed on historically valid ground. Some guiding principles are indicated for dealing with conflicting source materials for such studies.
\end{abstract}

W

HEN I received the persuasive invitation to speak today on a problem of theory construction and of the logic of discovery, I noted particularly the request to bring out the historical-sociological aspects. This directive was a pleasant surprise, for I recalled that Hans Reichenbach had flatly declared himself for the opposite view when he said "The philosopher of science is not much interested in the thought processes which lead to scientific discoveries..., that is, he is not interested in the context of discovery, but in the context of justification". ${ }^{1}$ If, therefore, I shall make some remarks on the origins of Einstein's special theory of relativity, I will be disobeying the Reichenbachian dictum. However, I draw further strength for this resolution from Einstein, who himself declared for the value of the historical treatment of the rise of key theories in science.

In fact, it is appropriate to say at the very outset to an audience consisting primarily of philosophers of science that sound historical investigations have lately perhaps been overlooked as important bases of sound philosophical discussions. Some examples come to mind immediately. The crux of the Copernican revolution was initially not, as is maintained in some philosophical works, a pragmatic search for the smallest num-

\footnotetext{
* Presented at the Symposium Theory Construction in Logical and Historical Perspective on December 27, 1959, organized by Section L (History and Philosophy of Science) of the AAAS, the American Philosophical Association, and the Philosophy of Science Association. Based on work-inprogress, supported in part by a grant of the National Science Foundation.

1 P. A. Schilpp, editor, Albert Einstein: PhilosopherScientist (Library of Living Philosophers, 1949), p. 292.
}

ber of components with which to build a world system, nor was it the establishment of the possibility of relativism in the choice of coordinate systems. Rather, as historians of science have shown, it was a return to an earlier, even an Aristotelian austerity concerning the type of motion judged to be suitable for the construction of the world system, mixed with a commitment to a neo-Platonic epistemology that looked for the warrant of reality in a new direction. The importance of Kepler is not that he was a mystic, an obsessed searcher for empirical rules, or a master of the intuitive, "personal" way to scientific knowledge; on the contrary, it can be shown that he was the first of the modern mathematical physicists, the first to look with some success for one dynamical explanation of all celestial and terrestial motions. Galileo, we have had to relearn only recently, was not the patron saint of laboratory experimentation, as philosophers of science have at times maintained. Concerning the abuse to which Newton's work has had to lend itself, the less said the better.

Einstein's work has not been immune from this fate. I am suggesting that in this case, as in the others, we build our philosophical analyses of science on real ground instead of dubious models, that we examine what physics was like in Einstein's time, what he did and said, how he came to do and say these things, and how he changed his mind-not once, but often. I urge this not as an easy program - for it is not that - and even not just because it is in principle better to do justice to the work of a man on his own terms rather than to use his work for a purpose which 
may have been inherently foreign to him. I urge this, rather, because I believe that a future source of strength of scholarship in the philosophy of science lies in philosophical analysis of historical cases.

I speak of Einstein's work because his case is both typical and special. The rise of relativity theory shares many features with the rise of other important scientific theories in our time, and in addition it is of course very much more: To find another work that illuminates as richly the relationship between physics, mathematics, and epistemology, or between experiment and theory, or one with the same range of scientific, philosophical and general intellectual implications, one would have to go back to Newton's Principia. The theory of relativity was a key development, both in physical science itself and also in modern philosophy of science. The reason for its dual significances is that Einstein's work provided not only a new principle of physics, but, as A. N. Whitehead said, "a principle, a procedure, and an explanation." Accordingly, the commentaries on the historical origins of the theory of relativity have tended to fall into two classes, each having distinguished proponents: the one views it as a mutant, a sharp break with respect to the work of the immediate predecessors of Einstein; the other regards it as an elaboration of then current work, e.g., by Lorentz and Poincaré.

To my mind, the Einsteinian innovation is understood best by superposition of both views, by seeing the discontinuity of methodological orientation within an historically continuous scientific development. ${ }^{2}$ Before we come to discuss this, and if we take seriously my point of view, we should first be ready to investigate a number of real problems of the historical or even "historicalsociological" kind: What are the sources for a study of the origins of the special theory of relativity $(R T)$ and what is their probable reliability? What was the state of science around 1905 , what were the contributions which prepared the field for the $R T$, and what did Einstein know about them? What were the steps by which Einstein reached the conclusions he published in 1905 ? To what extent was this work a member of

2 G. Holton, IX Congreso Internacional de Historia de las ${ }^{2} \mathrm{G}$. Holton, IX Congreso Internacional de Historia de las Madrid, 1959), Vol. II, p. 41. a continuous chain having as its immediate predecessors Lorentz and Poincaré? What was the role of experiment in the genesis of the $R T$, and what the role of the existence of contradictory hypotheses? What part played epistemological analysis in Einstein's thought? What was the early reception of the $R T$ among scientists? In particular, what was Einstein's relation with Mach, Lorentz, and Planck? What may we say about the style of Einstein's work and his personal orientations? What, if anything, in the origins and content of the $R T$ is typical of other theories with great impact on science? And even, what methodological principles for the study of the history of science emerge from this study?

We would find that the existing literature is not always of help in studying such questions. The literature on the $R T$ is of course vast. LeCat ${ }^{3}$ listed over 3400 scientific papers in the field up to 1922, with an approximately exponential growth giving a sevenfold increase in seven years. Biographically or philosophically oriented analyses are also fairly numerous (for example, by Schlick, Reichenbach, Frank, Meyerson, Cassirer, Whitehead, Wenzel, Grünbaum, Polanyi, Margenau, Lenzen, Bridgman, and Northrop.) It may be remarked there has so far been no full-scale historical study (although one is now in progress). A number of valuable essays exist in this direction (for example, by Born, Dugas, Kuznetsov, von Laue, Pauli, Straneo, and Whittaker); these are generally concerned with the chronological development of physics, and typically constitute a portion of a longer work having a purpose different from that of a primarily historicalphilosophical study. For the latter, the best source is at present indeed Einstein's own set of papers.

\section{CONTINUITY IN EINSTEIN'S WORK}

To these papers we must turn to discover, for example, the elements of continuity linking Einstein's first publication on the $R T$ with his other work at the time and with the older tradition itself. After the paper of $1905,{ }^{4}$ Einstein returned to the exposition of the $R T$ several times, and each restatement is of interest. For instance, in

\footnotetext{
3 Maurice LeCat, Bibliographie de la Relativité (Bruxelles, 1924).

${ }^{4}$ A. Einstein, Ann. Physik 17, 891 (1905).
} 
his book Über die spezielle und die allgemeine Relativitätstheorie ${ }^{5}$ he emphasized in his introduction that "the author has made the greatest effort to present the main ideas... on the whole in the sequence and in such context as they in fact arose." It is not surprising that the sequence given there is not in accord with the sequence of steps in the 1905 paper itself, but the historian of science finds an interesting problem in the fact that neither of these is in accord with other autobiographical or biographical accounts.

When one studies the relativity papers in the larger contextual setting of Einstein's other scientific papers, particularly those on the quantum theory of light and on Brownian motion which also were written and published in 1905, one notices two crucial points. While the three epochal papers of 1905-sent to the Annalen der Physik at intervals of less than eight weeksseem to be in entirely different fields, closer study shows that they arose in fact from the same general problem, namely, the fluctuations in the pressure of radiation. In 1905, as Einstein later wrote to von Laue, ${ }^{6}$ he had already known that Maxwell's theory leads to the wrong prediction of the motion of a delicately suspended mirror "in a Planckian radiation cavity." This connects on the one hand with the consideration of Brownian motion as well as to the quantum structure of radiation, and on the other hand with Einstein's more general reconsideration of "the electromagnetic foundations of physics" itself. ${ }^{7}$

One also finds that the style of the three papers is essentially the same, and reveals what is typical of Einstein's work at that time. Each begins with the statement of formal asymmetries or other incongruities of a predominantly esthetic nature (rather than, for example, a puzzle posed by unexplained experimental facts), then proposes a principle-preferably one of the generality of, say, the second law of thermodynamics, to cite Einstein's repeated analogy - which removes the asymmetries as one of the deduced consequences, and at the end produces one or more experimentally verifiable predictions.

Specifically, Einstein's first paper on the quan-

\footnotetext{
5 A. Einstein, (Braunschweig, 1916).

'Letter of January 17, 1952 (unpublished). See also Max Born in Funfaig Jahre Relativitätstheorie, edited by A. Mercier and M. Kervaire (Bern, 1955), pp. 248-249.

${ }^{7}$ See footnote reference 1 , p. 47 .
}

tum theory of light opens in a typical manner: "There exists a radical formal difference between the theoretical representations which physicists have constructed for themselves concerning gases and other ponderable bodies on the one hand, and Maxwell's theory of electromagnetic processes in so-called empty space on the other hand." 8 The significant starting point is a formalistic difference between theoretical representations in two fields of physics which, to most physicists, were so widely separated that no such comparison would have invited itself and therefore no such discrepancy would be noted. The discrepancy Einstein points out is between the discontinuous or discrete character of particles and of their energy on one hand, and the continuous nature of functions referring to electromagnetic events and of the energy per unit area in an expanding wave front on the other hand. The discussion of the photoelectric effect, for which this paper is mostly remembered, occurs toward the end, in a little over two pages out of the total sixteen. The prescription for obtaining an experimental verification of his point of view is given in a single, typically succinct Einsteinian sentence (straightline relation with constant slope between frequency of light and stopping potential for all electrode materials).

In his second paper published in $1905,{ }^{9}$ Einstein points out in the second paragraph that the range of application of classical thermodynamics may be discontinuous even in volumes large enough to be microscopically observable. He ends with the equation giving Avogadro's number in terms of observables in the study of particle motion, and with the one-sentence exhortation: "May some investigator soon succeed in deciding the question which has been raised here, and which is important for the theory of heat!" Significantly, Einstein reported the following year ${ }^{10}$ that only after the publication of this paper was his attention drawn to the experimental identification, as long ago as 1888, of Brownian motion with the effect whose existence he had deduced as a necessity from the kinetic-molecular theory. In his autobiographical notes he repeats that he did the work of 1905 "without knowing that observa-

\footnotetext{
${ }^{8}$ A. Einstein, Ann. Physik 17, 132 (1905).

9 A. Einstein, Ann. Physik 17, 549 (1905).
${ }^{10}$ A. Einstein, Ann. Physik 19, 371 (1906).
} 
tions concerning Brownian motion were already long familiar". ${ }^{11}$

The third paper of $1905^{12}$ is, of course, Einstein's first paper on the $R T$. He begins again by drawing attention to a formal asymmetry, i.e., in the description of currents generated during relative motion between magnets and conductors. The paper does not invoke explicitly any of the several well-known experimental difficultiesand the Michelson and Michelson-Morley experiments are not even mentioned when the opportunity arises to show in what manner the $R T$ accounts for them. At the end, Einstein briefly mentions here, too, specific predictions of possible experiments (giving the equation "according to which the electron must move in conformity with the theory presented here"). ${ }^{13}$

\section{RETURN TO A CLASSIC RESTRICTION ON FYPOTHESES}

The recognition of these common elements in the three papers prepares us for the essential realization that the fundamental postulates appearing in each of the three papers are heuristic. The heuristic nature of the postulate of relativity was from the beginning apparent to Einstein (as he asserted in 1907 and later) because of the restriction of the $R T$ to translational motions and to gravitation-free space..$^{14}$

The study of the three papers together reveals also the extent to which Einstein's $R T$ represents an attempt to restrict hypotheses to the most general kind and the smallest number possible-a goal on which Einstein often insisted. ${ }^{15}$ In the 1905 paper on $R T$, he makes, in addition to the

${ }^{11}$ See footnote reference 1, p. 47. See also L. Infeld, Albert Einstein (New York, 1950), p. 97-98.

${ }_{12}$ A. Einstein, Ann. Physik 17, 891 (1905).

${ }_{13}$ See footnote reference 12, p. 921.

${ }^{14}$ On a few occasions, although not in the original paper, Einstein made this point [e.g., Ann. Physik 23, 206 (1907) ]: "The relativity principle [is to be regarded].. solely as a heuristic principle, which, considered by itself, contains only assertions about rigid bodies, clocks, and light signals."

${ }^{15}$ Cf. A. Einstein, "The Problem of Space, Ether, and the Field in Physics," Ideas and Opinions by Albert Einstein, translated and revised by Sonja Bargmann (New York, 1954), p. 282: "The theory of relativity is a fine example of the fundamental character of the modern development of theoretical science. The initial hypotheses become steadily more abstract and remote from experience. On the other hand, it gets nearer to the grand aim of all science which is to cover the greatest possible number of empirical facts by logical deductions from the smallest possible number of hypotheses or axioms." two "conjectures" raised to "postulates" (i.e., of relativity and of the constancy of light velocity) only four other hypotheses: one of the isotropy and homogeneity of space, the others concerning three logical properties of the definition of synchronization of watches. In contrast, $\mathrm{H}$. A. Lorentz's great paper which appeared a year before Einstein's publication ${ }^{16}$ and typified the best work in physics of its time-a paper which Lorentz declared to be based on "fundamental assumptions" rather than on "special hypotheses"- contained in fact eleven ad hoc hypotheses: restriction to small ratios of velocities $v$ to light velocity $c$; postulation $a$ priori of the transformation equations (rather than their derivation from other postulates); assumption of a stationary ether; assumption that the stationary electron is round; that its charge is uniformly distributed; that all mass is electromagnetic; that the moving electron changes one of its dimensions precisely in the ratio of $\left(1-v^{2} / c^{2}\right)^{\frac{1}{2}}$ to 1 ; that forces between uncharged particles and between a charged and uncharged particle have the same transformation properties as electrostatic forces in the electrostatic system; that all charges in atoms are in a certain number of separate "electrons"; that each of these is acted on only by others in the same atom; and that atoms in motion as a whole deform as electrons themselves do. It is for these reasons that Einstein later maintained that the $R T$ grew out of the Maxwell-Lorentz theory of electrodynamics "as an amazingly simple summary and generalization of hypotheses which previously have been independent of one another..."."17

If one has studied the development of scientific theories, one notes here a familiar theme: the socalled scientific "revolution" turns out to be at bottom an effort to return to a classical purity. This is not only a key to a new evaluation of Einstein's contribution, but indicates a fairly general characteristic of great scientific "revolutions." Indeed, while it is usually stressed that Einstein challenged Newtonian physics in fundamental

${ }^{16}$ H. A. Lorentz, Proc. Acad. Sci. Amsterdam 6, 809 (1904). This paper, originally presented as part of the proceedings of the meeting of April 23, 1904, was first published in June, 1904 in the Dutch language edition of the Proceedings [12, 986-1009 (1904)].

${ }_{17}$ See footnote reference 5, p. 28. See also A. Einstein, Scientia 15, 338 (1914). 
ways, the equally correct but neglected point is the number of methodological correspondences with earlier classics, for example, with the Principia.

Here a listing of some main parallels between the two works must suffice: the early postulation of general principles which in themselves do not spring directly from experience; the limitation to a few basic hypotheses ${ }^{18}$; the exceptional attention to epistemological rules in the body of a scientific work; the philosophical eclecticism of the author; his ability to dispense with mechanistic models in a science which in each case was dominated at the time by such models ${ }^{19}$; the small number of specific experimental predictions; and the fact that the most gripping effect of the work is its exhibition of a new point of view.

The central problem, moreover, is the same in both works: the nature of space and time, and what follows from it for physics. Here, the basic attitudes have in both cases more in common than appears at first reading. That Newton's absolute space and absolute time were not meaningful concepts in the sense of laboratory operations, was, of course, not the original discovery of Mach; rather, it was freely acknowledged by Newton himself. But Einstein was also quite explicit that in replacing absolute Newtonian space and time with an infinite ensemble of rigid meter sticks and ideal clocks he was not proposing a laboratory-operational definition. He stated it could be realized only to some degree, "not even with arbitrary approximation," and that the fundamental role of the whole conception, both on factual and on logical grounds "can be attacked with a certain right." ${ }^{20}$ Thus the $R T$ merely shifted the locus of space time from the sensorium of Newton's God to the sensorium of Einstein's abstract Gedankenexperimenter-as it were, the final secularization of physics.

${ }^{18}$ Wolfgang Pauli, in Theory of Relativity [(B. G. Teubner, Leipzig, 1921 and Pergamon Press, New York, 1958), p. 5], unwittingly draws forceful attention to this particular point when summarizing his analysis of the $R T$ in the following words: "The postulate of relativity implies that a uniform motion of the center of mass of the universe relative to a closed system will be without influence on the phenomena in such a system." Note the correspondence with the main hypothesis in the last edition of the Principia.

${ }_{19}$ Cf. Max von Laue, Naturwissenschaften 43, 1 (1956).

${ }_{20}$ Les Prix Nobel en 1921-1922 (Stockholm, 1923), p. 2. See also A. Einstein, Naturwissenschaften 6, 692 (1918).
In his tribute on the occasion of the 200th anniversary of Newton's death, Einstein wrote: "I must emphasize that Newton himself was better aware of the weakness inherent in his intellectual edifice that the generation of learned scientists which followed him. This fact has always aroused my deep admiration...." ${ }^{21}$ He then immediately draws attention to the fact that "Newton's endeavors to represent his system as necessarily conditioned by experience and to introduce the smallest number of concepts not directly referable to empirical objects is everywhere evident." He recalls that Newton regarded the law of gravitational interaction as a heuristic device, "not supposed to be a final explanation, but a rule derived by induction from experience." When the essay ends with Einstein clearly associating himself with a view of causality which he characterizes as "Newtonian," he could well have widened the context of that remark.

\section{TIME-DEPENDENCE IN SOURCE MATERIALS}

I cannot avoid a word of warning on the use of sources such as Einstein's writings, particularly to an audience not professionally engaged in the study of the history of science. This has to do with the fact that in many important particulars the writings of one man do not by any means necessarily overlap. I am not speaking merely of the fact that Einstein regarded the discoverer, and particularly himself, as a very poor source of information concerning the genesis of his own ideas, and suggested rather that this study was one of the most interesting tasks for the historian of science. No, I have in mind the simple, yet often neglected fact that Einstein as a person with a single, unchanging identity, in a real sense never existed, just as there never was a single unchangeable entity called Galileo or Newton or Dalton. Einstein himself saw this clearly when he wrote at the start of his autobiographical notes ${ }^{22}$ :

The exposition of that which is worthy of communication does nonetheless not come easy; today's person of 67 is by no means the same as was the one of 50 , of 30 , or of 20 . Every reminiscence is colored by today's being what it is, and therefore by a deceptive point of view.... And it is not

${ }^{21}$ A. Einstein, Naturwissenschaften 15, (1927); reprinted in Ideas and Opinions of Albert Einstein (New York, 1954) 257 .

22 See footnote reference 1 , pp. 3-7. 
only growth or change-it is also the difference between experience lived and experience reported. In this case it is well possible that such an individual in retrospect sees a uniformly systematic development, whereas the actual experience takes place in kaleidoscopic particular situations.

These two effects, coupled with Einstein's large output of writings of both a scientific and a popular kind, explain why everyone-from the extreme positivist to the critical realist-can find some part of Einstein's work to nail to his mast as a battle flag against the others.

There are two ways of dealing with this problem in historically oriented work. The first is to be explicitly careful in the evaluation of all sources, including autobiographical statements, to allow a time-dependent weighting factor. This has always been true, but is particularly pertinent in modern physics where changes per unit time are far larger than before. Revealing examples, and very worthwhile topics of study, are Einstein's attitude to the ether problem, or his relation to Ernst Mach, or his more general epistemological position. Concerning the first of these, for instance, Einstein underwent a profound change of orientation between the statement near the beginning of his fundamental 1905 paper: "The introduction of a 'luminiferous ether' will prove to be superfluous inasmuch as the view here to be developed will not require an 'absolutely stationary space' provided with special properties"-a provocative remark on which Dugas astutely comments "such a declaration, made on the threshold of his theory, could only alienate him from the physicists imbued with the classical representation" 23 - to his Leiden speech of 1920 on "Äther und Relativitätstheorie" in which he says near the close: "Recapitulating, we may say that according to the general theory of relativity space is endowed with physical qualities ; in this sense, therefore, there exists an ether. According to the general theory of relativity, space without ether is unthinkable; for in such a space there not only would be no propagation of light, but also no possibility of existence for standards of space and time (measuring rods and clocks), nor therefore any space-time intervals in the physical sense." ${ }^{24}$

\footnotetext{
${ }^{23}$ René Dugas, A History of Mechanics (New York, 1955), p. 490.

24 In A. Einstein, Sidelights on Relativity (Methuen and Company, Ltd., London, 1922), p. 23. The next sentences
}

To the student of the nature of scientific theories, a sequence of individual documents on a particular topic from one pen represents therefore, as it were, a sequence of cross sections in space-time, from which he is challenged to reconstruct the progress or worldline of the topic. Particularly in recent and contemporary physics, no single segment of this worldline may be safely extrapolated; a quick turn is always likely. This enhances the interest: the reconstruction of the changing course of opinion on a topic becomes doubly important, and these changes in one topic may often be correlated with changes in another topic. In the case of Einstein, for example, the attitudes to the ether, to Mach, to epistemology and metaphysics generally, and to religion, all show closely correlated changes in time. This itself poses new and valuable problems, both to the historian and to the philosopher of science.

\section{THE COMPLEMENTARITY OF SOURCE MATERIALS}

There is a second problem involving divergent or contradictory views concerning a scientist's work. It is generated not by internal changes or conflicts, but by external ones. I can discuss this in the briefest way by pointing to the question of what one is to do with biographical works which are not in agreement.

Such biographies are a precious set of sources for the study of the origins of the relativity theory. Among the principal ones that appeared in Einstein's own time are, in order of publication, those by Moszkowski, Reiser, Reichinstein, Marinoff and Wayne, Seelig, Frank, Infeld, and Vallentin. Each has interest in its own right, but naturally enough they differ vastly in their points of view as well as on factual matters. One can begin to discern the Vivianis and Stukleys now, the sources of future myths and the sources of

reaffirm the difference between this and other ether models. "But this ether may not be thought of as endowed with the quality characteristic of ponderable media, as consisting of parts which may be tracked through time. The idea of motion may not be applied to it."

In this connection, see also the essay on "Relativity and the Problem of Space," which Einstein added in the 16th edition of Relativity, the Special and General Theory (Methuen and Company, Ltd., London, 1952). Commenting on it to Carl Seelig [Albert Einstein (Zürich, 1954), p. 291], Einstein wrote: "In particular, it is shown that the development has a close connection with Descartes' argument for the non-existence of "empty space'." 
reliable references. It was therefore important to discover the unpublicized fact that one of these was written under a pseudonym by a relative of Einstein and checked by him for factual accuracy, that another was publicly disowned by Einstein, that he made an attempt in a third case to persuade the author-whom he did not trust to be fair or accurate - to forgo publication, that he was pleased with the material in another of these books, and so forth.

The uncommonly large amount and variety of material emphasizes the problems the historian of science must face. The different points of view from which two or more honest biographies are written yield, of course, different interpretations. On some matters of "fact" (as, for example, dates and places) one certainly can ask for agreement or accuracy in some absolute sense. But on larger and more qualitative questions (for example, the acceptance of the theory) one can profitably adopt the attitude that evidence obtained by biographical research under different points of view cannot be comprehended within a single picture, but must be regarded as complementary in the sense that only the totality of the presentations exhausts the possible information about the subject. This will be recognized as closely analogous to one part of the complete statement of the complementarity principle in physics. ${ }^{25}$ To look for an "independent" view in qualitative matters in any other way is likely to lead one to take merely a position equidistant between all others, or between the "isms" that motivate them.

The complementarity principle tells the physicist also that it is not possible to make a sharp separation between the behavior of atomic objects and the interaction with the measuring instruments which serve to define the conditions under which the phenomena appear. This statement, too, has a close parallel in the study of the history or philosophy of science, and one must therefore be aware that the scholar and the subject of his study together form one system in which it is not meaningful to try to achieve a complete separation of one part from the other. It is in this spirit that one must understand, and use, the picture of Einstein as a revolutionary which is painted by a revolutionary, and that of

\footnotetext{
${ }^{25} \mathrm{I}$ employ it here as a suggestive, though not prescriptive, analogy.
}

Einstein as a positivist as presented by a positivist. To one who is committed to the existence of a real medium to explain the transmission of light through space, the $R T$ is important primarily insofar as it adds to or subtracts from this position. Only with this explicit recognition can one use a number of accounts together, each of which would otherwise appear to present a strikingly different person or work.

\section{WHITTAKER'S ACCOUNT OF THE ORIGINS OF EINSTEIN'S WORK}

To illustrate this point concretely I wish to turn to a question on which a dispute has been active, namely, to what extent Einstein's work was original rather than anticipated by, or specifically based on, other published work. Particularly interesting is the essay on Einstein by Sir Edmund Whittaker in the Biographical Memoirs of Fellows of the Royal Society (London, 1955). Whittaker's commitment to the 19th-century tradition of physics and to the ether theory is illustrated in his well-known book $A$ History of the Theories of Aether and Electricity up to about 1900 (London, 1910; 2nd ed., 1951), and also by his excellent contributions in the field of classical mechanics. Moreover, in the second volume of the History, completed in 1953, which carries the story to 1926 , Whittaker had largely dismissed Einstein's paper of 1905 on the RT as one "which set forth the relativity theory of Poincaré and Lorentz with some amplifications, and which attracted much attention." 26

This presentation evoked considerable criticism, some of which I know to have reached Whittaker while his book was still in manuscript, and some of which reached him by the time he composed the biographical memoir after Einstein's death in 1955. It is therefore noteworthy that in his 1955 necrolog for Einstein, Whittaker has not changed his earlier evaluation. For example, he repeats that Poincaré in a speech in St. Louis, U. S. A., in September 1904 27 had coined the phrase "principle of relativity." Whittaker asks how physics could have been reformu-

${ }^{26}$ Sir Edmund Whittaker, A History of the Theories of Aether and Electricity: The Modern Theories 1900-1926 (New York, 1954), p. 40.

${ }_{27}$ J. H. Poincaré, Bull. Sci. Math. (1904); English translation in Monist, 15, 1 (1905). 
lated in accordance with "Poincaré's principle of relativity," and he reports that with respect to the laws of the electromagnetic field this "discovery was made in 1903 by Lorentz," citing a paper by Lorentz in the Proceedings of the Academy of Sciences, Amsterdam, for the year 1903. ${ }^{28}$ Whittaker shows that "the fundamental equations of the aether in empty space" are invariant under suitably chosen (i.€.. Lorentz) transformations, and he concludes with the remarkable sentence: "Einstein in [the $R T$ paper of 1905] adopted Poincaré's principle of relativity, using Poincaré's name for it, as a new basis for physics and showed that the group of Lorentz transformations provided a new analysis connecting the physics of bodies in motion relative to each other." ${ }_{29}$

Since Whittaker's analysis has been and is likely to continue to be given considerable weight, it is necessary to examine it closely. It turns out to be an excellent example of the proposition that no such analysis can be considered meaningful except insofar as it deals both with the material it purports to cover and with the prior commitments and prejudices of the scholar himself. Here is a brief summary of main findings when Whittaker's analysis is considered in this light.

(1) Einstein's RT paper of 1905 was indeed one of a number of contributions by many different authors in the general field of the electrodynamics of moving bodies. In the Annalen der Physik alone there are eight papers from 1902 up to 1905 concerned with this general problem. Einstein himself always insisted on this aspect of continuity. The earliest evidence is in a letter written in the spring of 1905 to his friend Conrad Habicht, describing his various investigations. In one sentence he describes the developing $R T$ paper: "The fourth work lies at hand in concept [liegt im Konzept vor] and is an electrodynamics of moving bodies making use of a modification of the theory of space and time; you will surely be interested in the purely kinematic part of this work." (In Carl Seelig, see footnote reference 24, p. 89. Ital. suppl.) Seelig (ibid., p. 97) also quotes a later remark of Einstein which gives in one

\footnotetext{
${ }^{28}$ The citation given is "Proc. Acad. Sci. Amst. (English ed.) (1903) 6, 809."

${ }_{29}$ Sir Edmund Whittaker, in Biographical Memoirs of

Fellows of the Royal Society (London, 1955), p. 42.
}

sentence his often repeated attitude: "With respect to the theory of relativity it is not at all a question of a revolutionary act, but of a natural development of a line which can be pursued through centuries."

On the other hand, to say that Einstein's paper "attracted much attention" is correct only if one neglects the first few years after publication. For the early period, a more characteristic reaction was, in fact, either total silence or the response to be found in the first paper in the Annalen der Physik that mentioned Einstein's work on the $R T$. It was a categorical experimental disproof of Einstein's theory by the eminent physicist W. Kaufmann, who concluded ${ }^{30}$ :

"I anticipate right here the general result of the measurements to be described in the following: The measurement results are not compatible with the Lorentz-Einsteinian fundamental assumption."

(2) The paper by Poincaré of 1904 which Whittaker cites turns out not to enunciate the new relativity principle, but is rather a very acute and penetrating though qualitative summary of the difficulties which contemporary physics was then making for six classical laws or principles, including what is in effect the Galilean-Newtonian principle of relativity. The list given by Poincaré is as follows: The law of conservation of energy; the second law of thermodynamics; the third law of Newton; "the principle of relativity, according to which the laws of physical phenomena should be the same whether for an observer fixed or for an observer carried along in a uniform movement or translation..."; the principle of conservation of mass; and the principle of least action. ${ }^{31}$ Of the principle of relativity Poincare complains that it "is battered" by current developments in electromagnetic theory, although, he says, it "is confirmed by daily experience" and "is imposed in an irrestible way upon one's good sense." Poincaré's main point is to show the need for a new development, the outlines of which he suggests in these words: "Perhaps likewise we should construct a whole new mechanics, that we only succeed in catching a glimpse of, where inertia increasing with the velocity, the velocity of light would become an

\footnotetext{
so W. Kaufmann, Ann. Physik 19, 495 (1906). Italics in original.

${ }^{31}$ See footnote reference 27, p. 5 .
} 
impassable limit." 32 Thus he illustrates both the power of his intuition and the qualitative nature of the suggestion.

(3) It is more difficult to discuss the 1903 paper of Lorentz which Whittaker, both in his book and in his Memoir, cited specifically as the work that spelled out most of the basic details of Einstein's $R T$ of 1905. In the first place, this paper does not exist. What Whittaker clearly wished to refer to is the paper Lorentz published a year later, in $1904 .{ }^{16}$ Since Whittaker was otherwise very careful with the voluminous citations of references, this repeated slip, which doubles the time interval between the work of Lorentz and of Einstein, is not merely a mistake. It is at least a symbolic mistake - symbolic of the way a biographer's preconceptions interact with his material.

(4) Whittaker clearly implied that Einstein used Lorentz's transformation equation published in 1904. He therefore chose to neglect that both Einstein and those close to him have repeatedly said that Einstein had not read Lorentz's 1904 paper. ${ }^{33}$

(5) Even if one does not wish to rely on the word of Einstein and other prominent physicists of his time in this matter, there are four items of internal evidence in Einstein's 1905 paper which indicate that he had not read Lorentz's of 1904. Einstein does write the transformation equations in a form equivalent to those of Lorentz (or, for that matter, of Voigt's of 1887); but whereas Lorentz had assumed these equations $a$ prior $i$ in order to obtain the invariance of Maxwell's equations in free space, Einstein derived them from the two fundamental postulates of the $R T$. He therefore did not need to know of Lorentz's paper of $1904 .^{34}$

\footnotetext{
${ }^{32}$ See footnote reference 27, p. 23 .

33 See the footnote on this point by. A. Sommerfeld in the reprints and translations of Einstein's 1905 paper in the Teubner and Methuen editions of the collection of essays on the RT [e.g., The Principle of Relativity (London, 1923) ]; or Pauli (footnote reference 18, p. 3) ; or Einstein's letter to Carl Seelig: "As for me, I knew only Lorentz's important work of 1895 . . but not Lorentz's later works and also not the inquiries of Poincaré connected with them. In this sense my work of 1905 was independent." [Techn. Rundschau, Bern, 47, (May 6, 1955); cited in Max Born, footnote reference 6 , p. 248 .]

${ }^{34}$ 'This is by no means the only such case in Einstein's early scientific career. In fact, his work on thermodynamics and fluctuation phenomena in the period 1902-1905 was to a large extent a repetition of available material; as Einstein said later, "Not acquainted with the earlier investigations
}

Secondly, as Einstein's first two major papers of 1905 show, he was in the habit of giving credit in footnotes to the work of others which he might be using; the absence of a specific reference to the 1904 paper of Lorentz may therefore be taken at its face value, the more so since Einstein twice in the text of this same paper refers to Lorentz by name in citing the then current electromagnetic theory in the form Lorentz had given it in his book of $1895 .^{35}$ Parenthetically one may also say that it is rather preposterous to suggest that a young man of Einstein's temperament and painful honesty, and one who, as the letters to Lorentz soon thereafter show, revered Lorentz deeply, should knowingly be using, without acknowledgment, an important new finding in the recent work of the foremost theoretical physicist in this field..$^{36}$

Next, in the second paragraph of his paper, Einstein recalls that the "laws of electrodynamics and optics" have been found to "be valid for all frames of reference for which the equations of mechanics hold good" to the first order of the quantity $v / c$. But one of the main points of the 1904 paper of Lorentz was his claim to have extended the theory to the second order of $v / c$. And a fourth internal evidence is Einstein's choice of convention in the expression for force and mass in the dynamics of charged particles; this choice ${ }^{37}$ is far less suitable than Lorentz's, forcing Planck to point this out in 1906.

(6) Quite apart from the question whether Einstein's 1905 paper was written independently

of Boltzmann and Gibbs, which had appeared earlier and actually exhausted the subject, I developed the statistical mechanics and the molecular-kinetic theory of thermodynamics which was based on the former." (P. A. Schlipp, footnote reference 1 , p. 47). Einstein's unawareness in 1905 of the earlier identification of Brownian motion has been referred to previously. Anton Reiser provides the report [in Albert Einstein (New York, 1930), p. 52] that at his university Einstein planned to build a device for measuring the ether drift, not knowing of Michelson's apparatus; although this earliest example is quite understandable in terms of the incompleteness of Einstein's training at that point, it illustrates a remark made often about him by his friends: that he read little, but thought much.

${ }^{35} \mathrm{H}$. A. Lorentz, Versuch einer Theorie der elektrischen und optischen Erscheinungen in bewegten Körpen (Leiden, 1895).

${ }^{36}$ Einstein later accurately reported that "At the turn of the century, H. A. Lorentz was regarded by theoretical physicists of all nations as the leading spirit; and this with fullest justification." A. Einstein, in H. A. Lorentz, edited by G. L. de Haas-Lorentz (Amsterdam, 1957), p. 5.

${ }_{37}$ As most recently remarked by Max von Laue [Naturwissenschaften $43,4(1956)]$ in documenting his belief that Einstein did not know of Lorentz's 1904 paper. 
of Lorentz's is the equally significant fact that in a crucial sense Lorentz's paper was of course not on the relativity theory as we understand the term since Einstein. Lorentz's fundamental assumptions are not relativistic; as Born says, "he never claimed to be the author of the principle of relativity," ${ }^{38}$ and, on the contrary, referred to it as "Einstein's Relativitätsprinzip" in his lectures of 1910. In Lorentz's essay on "The Principle of Relativity of Uniform Translation," published in 1922, ${ }^{39}$ six years before Lorentz's death, he still asked that space be considered to have "a certain substantiality; and if so, one may, in all modesty, call true time the time measured by clocks which are fixed in this medium, and consider simultaneity as a primary concept." 40 In his 1904 paper he had postulated the nonrelativistic addition theorem for velocities, $v=V+u$, and even in the 1922 book he did not consider the velocity of light as inherently the highest attainable velocity of material bodies.

(7) Finally, we note another set of important differences between Lorentz's accomplishment of 1904 and what Whittaker implies. Strictly speaking, the Lorentz theory of 1904 applies only to

${ }_{38}$ Max Born, footnote reference 6, p. 247.

${ }^{39}$ A. D. Fokker, editor, translated as Vol. III of Lectures on Theoretical Physics (London, 1931).

${ }_{40}$ See footnote reference 39, p. 211. small values of $v / c$, since the constant $l$ which is taken to be 1 for small values of $v / c$ enters in the first power in the transformation equations for $x$ and $t$. Also, Maxwell's equations in the presence of charges are not completely invariant in Lorentz's treatment even at small speeds $v$, since in the primed (moving) system, a term is left over in the expression for $\operatorname{div}^{\prime} D^{\prime}$, namely, $\operatorname{div}^{\prime} D^{\prime}$ $=\left[1-\left(v u_{x}^{\prime} / c^{2}\right)\right] \rho^{\prime}$, as compared to $\operatorname{div} D=\rho .^{41}$ We have already noted the number of ad hoc hypotheses which Lorentz was forced to introduce, and which, Einstein felt, robbed the theory of electromagnetic phenomena of the generality typical of fundamental conceptions.

In closing, I return to my initial remarks: The detailed study of the historical situation is, to my mind, an important first step in those discussions which try to base epistemological considerations on "real" cases. This is not always done easily; but it is through the dispassionate examination of historically valid cases that we can best become aware of the preconceptions which underlie all philosophical study.

${ }^{41}$ Whittaker (footnote reference 26, p. 31), says Lorentz "obtained a transformation in a form which is exact to all orders of the small quantity $v / c$," although strictly speaking this is correct only for free space and relatively small values of $y$. 